\title{
Valoración global del corazón en el paciente con transplante cardiaco mediante tomografía computarizada de doble fuente
}

\author{
Global evaluation of the heart in heart transplant recipients using \\ dual-source computed tomography
}

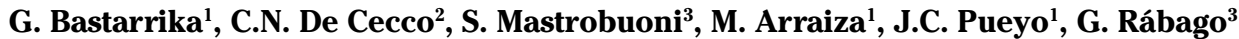

\section{RESUMEN}

La ecocardiografía y la coronariografía convencional son las dos técnicas empleadas en la práctica clínica para el seguimiento de los pacientes con transplante cardiaco. La coronariografía permite diagnosticar la vasculopatía del injerto, pero se trata de un procedimiento invasivo no exento de complicaciones. La tomografía computarizada multicorte convencional es una técnica no invasiva útil para descartar enfermedad coronaria y estudiar la función cardiaca. Sin embargo, debido a su resolución temporal es necesario administrar fármacos betabloqueantes por lo que su utilidad en los pacientes con escasa respuesta a esta medicación, como los transplantados cardiacos, es limitada. La TC de doble fuente permite estudiar las arterias coronarias en todos los individuos con independencia de su frecuencia cardiaca. En el caso que se presenta se demuestra, además, que puede ser una técnica útil para valorar la función y descartar la vasculopatía del injerto en los pacientes con transplante cardiaco.

Palabras clave. Corazón. Vasos coronarios. Tomografía computarizada. Transplante cardiaco.

\begin{abstract}
In routine clinical practice surveillance of heart transplant recipients is usually performed using echocardiography and conventional coronary angiography. The latter permits diagnosis and follow-up of coronary allograft vasculopathy. However, this procedure is invasive and is not free of complications. Conventional multislice computed tomography (MSCT) has been shown to be a useful non-invasive tool for ruling out coronary artery disease and evaluating cardiac function. However, due to its limited temporal resolution betablocker administration is required, and its usefulness in certain patient populations with restricted response to this medication, such as heart transplant recipients, may therefore be limited. Dual-source CT (DSCT) allows evaluation of the coronary arteries in all individuals independent of their heart rate. In the case presented here, we demonstrate that DSCT may be useful for evaluating cardiac function and ruling out coronary allograft vasculopathy in heart transplant recipients.
\end{abstract}

Key words. Heart. Coronary vessels. Computed tomography. Heart transplantation.
1. Servicio de Radiología. Clínica Universitaria. Universidad de Navarra.

2. Servicio de Radiología. Universita' di Roma Sapienza-"Ospedale Sant'Andrea".

3. Departamento de Cirugía Cardiovascular. Clínica Universitaria. Universidad de Navarra.

Recepción el 2 de diciembre de 2008

Aceptación provisional el 15 de enero de 2009

Aceptación definitiva el 15 de enero de 2009
Correspondencia:

Gorka Bastarrika

Servicio de Radiología

Clínica Universitaria

Avda. Pío XII, 36

31008 Pamplona

Tfno. +0034948255400

Fax +0034948296500

E-mail: bastarrika@unav.es 


\section{INTRODUCCIÓN}

El transplante cardiaco es la única opción terapéutica que cambia significativamente la evolución natural de la insuficiencia cardiaca. España ocupa el segundo lugar en número de transplantes cardiacos por millón de habitantes y año por detrás de EE.UU. ${ }^{1}$.

El seguimiento de los pacientes con transplante cardiaco se realiza mediante la combinación de pruebas no invasivas e invasivas. Las técnicas no invasivas que habitualmente se utilizan para cuantificar los parámetros de función y masa ventricular del injerto son la ecocardiografía y la resonancia magnética, siendo esta última la técnica más precisa ${ }^{2}$. Entre los procedimientos invasivos destacan la coronariografía convencional y la ecografía intravascular, útiles para diagnosticar la vasculopatía del injerto, una entidad propia que influye significativamente en la evolución del corazón transplantado ${ }^{3}$ y que aunque con frecuencia es asintomática, sus manifestaciones clínicas pueden ser variables (arritmia, insuficiencia cardiaca o muerte). Estos dos procedimientos invasivos, especialmente en pacientes con transplante cardiaco, conllevan un riesgo no despreciable de complicaciones ${ }^{4}$.

La tomografía computarizada multicorte (TCMC) convencional es una técnica útil para descartar enfermedad coronaria ${ }^{5} \mathrm{y}$ determinar la función ventricular en una misma exploración ${ }^{6}$, aunque posee limitaciones especialmente en sujetos con frecuencias cardiacas elevadas. El recientemente introducido sistema de TC de doble fuente (TCDF) (Somatom Definition, Siemens Medical Solutions, Forchheim, Alemania) permite obtener imágenes de las arterias coronarias de excelente calidad diagnóstica con independencia de la frecuencia cardiaca ${ }^{7}$.

El caso que se presenta demuestra la posibilidad de realizar una valoración global del corazón en el paciente con transplante cardiaco en una sola apnea utilizando un equipo de TCDF. Se describe la posibilidad de estudiar el estado de las arterias coronarias, valorar la función del injerto y cuantificar la masa ventricular en un paciente con transplante cardiaco al que se le realizó un estudio cardiaco mediante TCDF para descartar vasculopatía del injerto.

\section{CASO CLÍNICO}

Paciente varón de 77 años no alérgico, exfumador, con antecedentes personales de miocardiopatía dilatada de origen enólico, motivo por el que se le realizó un trasplante cardíaco ortotópico hace nueve años. En grado funcional NYHA I desde la última revisión anual. El electrocardiograma mostró ritmo sinusal normal a 95 latidos por minuto (lpm), signos de dilatación de aurícula izquierda y bloqueo bifascicular con bloqueo de rama derecha y hemibloqueo de rama izquierda. En la radiografía de tórax se observaron suturas de esternotomía media, ateromatosis a nivel de cayado y silueta cardiovascular dentro de límites normales. Se describió un tórax discretamente hiperinsuflado que sugería enfisema pulmonar con prominencia hiliar bilateral que parecía vascular. En el estudio ecocardiográfico transtorácico se evidenció que la función sistólica global y regional del corazón transplantado se encontraban conservadas (fracción de eyección, FE: 0,61). Se estimó un volumen telediastólico (VTD) de $73 \mathrm{ml}$, volumen telesistólico (VTS) de 28 ml y masa miocárdica de 93 gramos. El patrón de llenado ventricular era el propio del corazón trasplantado. No se objetivaron signos de elevación de la presión de llenado. La ecocardiografía de estrés con dobutamina fue normal. El test de provocación de isquemia resultó negativo para isquemia miocárdica.

Con objeto de estudiar las arterias coronarias y descartar vasculopatía del injerto se realizó una coronariografía mediante TCDF con los siguientes parámetros: 120 kV, 350 mAs efectivos para cada tubo, CTDIvol= $41 \mathrm{mGy}$. Se administraron $70 \mathrm{ml}$ de contraste yodado (Iomeron 400, $400 \mathrm{mgl} / \mathrm{ml}$, Bracco, Milán, Italia) a través de una vena antecubital derecha. Durante la exploración la frecuencia cardiaca media del sujeto fue de $80 \mathrm{lpm}$ (rango 78-83 lpm). La duración de la apnea fue de ocho segundos. En esta exploración se objetivó dominancia izquierda con arterias coronarias normales (Fig. 1), sin estenosis significativas (Fig. 1). La arteria coronaria derecha era de calibre fino, sobre todo en sus tercios medio y distal (Fig. 1C, 1D). Como variante anatómica se observó un ramo intermedio o bisectriz (Fig. 1E). El cálculo de la función ventricular izquierda mediante TCDF mostró parámetros superponibles a los obtenidos con la ecocardiografía (Fig. 2). La función cardiaca se encontraba en límites normales con FE: 0,61, VTD $61 \mathrm{ml}$, VTS $24 \mathrm{ml}$ y masa miocárdica de 88 gramos. No se objetivaron anomalías de la contractilidad regional. Dado que la ecocardiografía de estrés fue negativa para isquemia miocárdica y la coronariografía no invasiva mediante TCDF mostró arterias coronarias epicárdicas normales, se desestimó la realización de coronariografía convencional. 


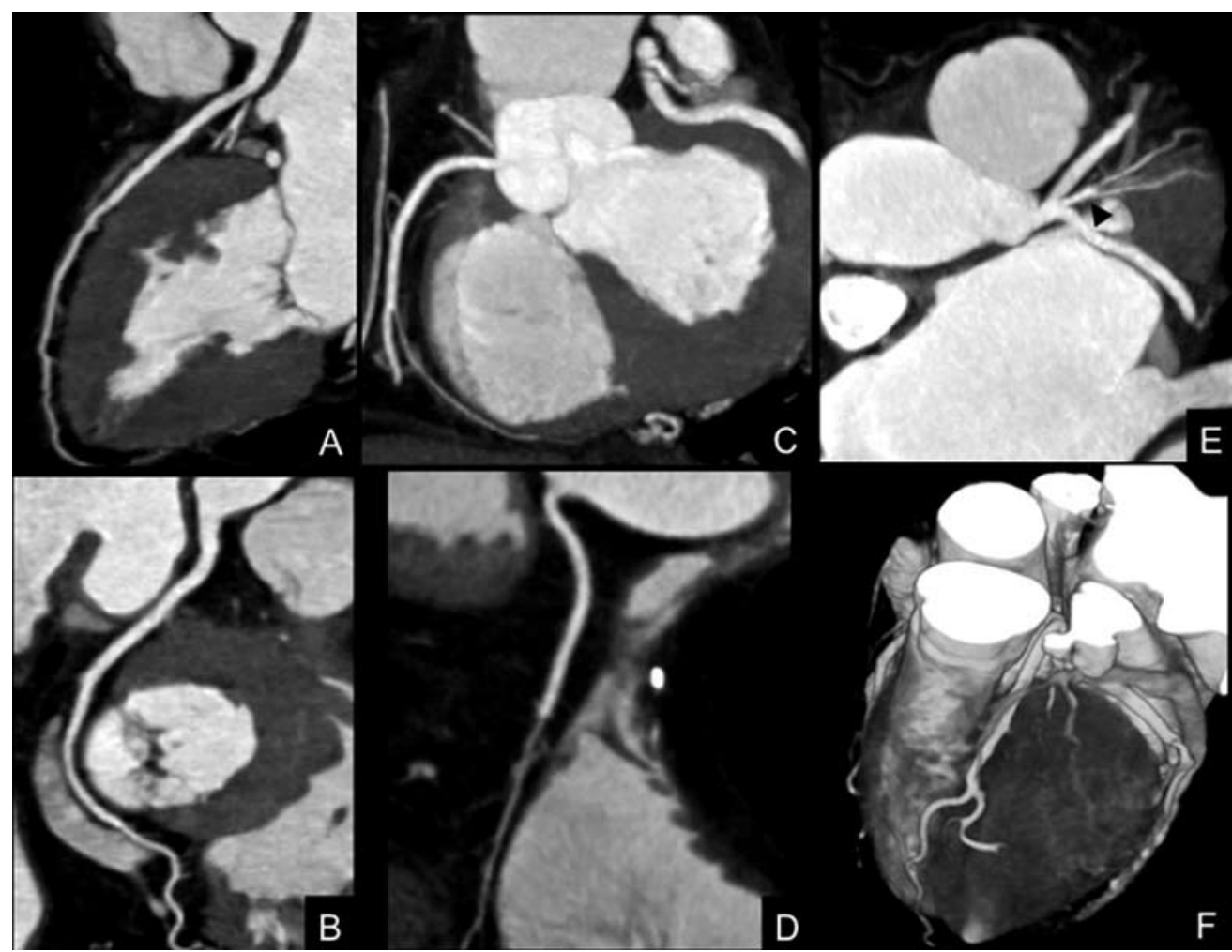

Figura 1. Coronariografía por TCDF de un sujeto con transplante cardiaco ortotópico. A. Reconstrucción curva de la arteria coronaria descendente anterior. B. Reconstrucción curva de la arteria coronaria circunfleja. C. Reconstrucción oblicua de la arteria coronaria derecha. D. Reconstrucción curva de la arteria coronaria derecha. E. Reconstrucción oblicua del ramo intermedio o bisectriz (punta de flecha). F. Reconstrucción volumétrica del árbol coronario. El estudio puso de manifiesto arterias coronarias normales con dominancia izquierda. Nótese el tamaño y la longitud de la arteria coronaria derecha (C, D) con respecto a la arteria circunfleja dominante (B).

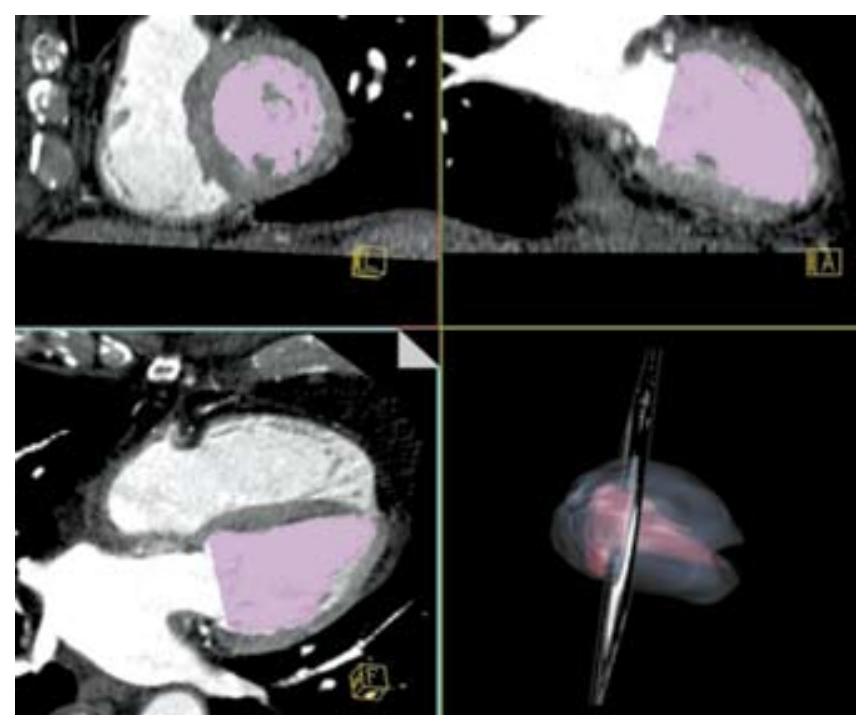

Figura 2. Coronariografía por TCDF. Interfaz de un software comercial de segmentación semiautomática (Circulation II, Siemens Medical Solutions) empleado para calcular la función ventricular. 


\section{DISCUSIÓN}

La vasculopatía del injerto es una de las causas más importantes de morbimortalidad en pacientes con transplante cardia$\mathrm{co}^{8}$. La coronariografía convencional es la técnica que se emplea en la práctica diaria para diagnosticar esta enfermedad ${ }^{9}$, una técnica invasiva no exenta de complicaciones. Dado que los pacientes con transplante cardiaco requieren la realización de cateterismos periódicos para valorar la vasculopatía del injerto, el desarrollo de una técnica que permitiera detectar esta enfermedad, en muchos casos silente, sería de gran utilidad para este grupo de pacientes.

La TCMC ha demostrado ser eficaz para descartar enfermedad coronaria en sujetos con sospecha de cardiopatía isquémica ${ }^{10} \mathrm{y}$ en pacientes con transplante cardiaco $^{11,12}$ siendo, además, una técnica útil para estudiar la función cardiaca en la misma exploración, sin necesidad de administrar mayor cantidad de contraste o radiación ${ }^{13}$. La utilidad de la coronariografía por TCMC radica en su alto valor predictivo negativo, porque estudios recientes subrayan la posibilidad de utilizar esta exploración para reemplazar el cateterismo convencional en determinados grupos de pacientes ${ }^{14}$.

Aunque se ha descrito la posibilidad de estudiar la vasculopatía del injerto con TCMC de 64 cortes, determinados factores como la limitada resolución temporal de estos equipos y características propias de los pacientes con transplante cardiaco como son su elevada frecuencia cardiaca, escasa respuesta a fármacos betabloqueantes, obesidad y cambios postquirúrgicos han restringido la implantación de la técnica para controlar este grupo de pacientes ${ }^{13}$.

La TCDF es un equipo multicorte de última generación cuya característica principal es que posee una resolución temporal constante de $83 \mathrm{~ms}$ (frente a los $165 \mathrm{~ms}$ de los equipos TCMC de 64 cortes). Esta característica permite que se puedan estudiar todos los sujetos, sin necesidad de administrar fármacos betabloqueantes, ya con que este equipo de TCMC se pueden obtener estudios de las arterias coronarias de gran calidad diagnóstica incluso con frecuencias cardiacas elevadas ${ }^{7}$. En los pacientes transplantados este equipo de TC también permite cuantificar la función ven- tricular izquierda con una exactitud casi superponible a la de la resonancia magnética $^{15}$.

Este caso se presenta como ejemplo de la posibilidad de realizar en una sola apnea de apenas ocho segundos una valoración global del injerto en el paciente con transplante cardiaco. Como se ha mostrado a pesar de la elevada frecuencia cardiaca del paciente durante la exploración (media $80 \mathrm{lpm}$; rango 78-83 lpm) se descartó la existencia de estenosis significativas en las arterias coronarias epicárdicas. La ecocardiografía de estrés no puso de manifiesto isquemia miocárdica inducible. Los resultados de ambas pruebas evitaron la realización de un cateterismo diagnóstico. Por tanto, el caso que se presenta muestra el potencial de combinar técnicas diagnósticas no invasivas para realizar una valoración global del injerto y evitar exploraciones cruentas para el diagnóstico y/o control evolutivo de la vasculopatía del injerto. En particular, dado su elevado valor predictivo negativo, la TCDF podría ser útil para descartar vasculopatía del injerto en los pacientes con transplante cardiaco evitando la necesidad de realizar cateterismo diagnósticos anuales para el seguimiento del injerto.

\section{BIBLIOGRAFÍA}

1. Alonso-Pulpon L. El trasplante cardiaco en España. Organización y resultados. Rev Esp Cardiol 2000; 53 (Suppl 1): 39-52.

2. Bellenger NG, Marcus NJ, Rajappan K, Yacoub M, Banner NR, PENnell DJ. Comparison of techniques for the measurement of left ventricular function following cardiac transplantation. J Cardiovasc Magn Reson 2002; 4: 255-263.

3. TAYlor DO, Edwards LB, Boucek MM, Trulock EP, KECK BM, HerTz MI. The Registry of the International Society for Heart and Lung Transplantation: twenty-first official adult heart transplant report-2004. J Heart Lung Transplant 2004; 23: 796-803.

4. Clague JR, Cox ID, Murday AJ, Charokopos N, MADDEN BP. Low clinical utility of routine angiographic surveillance in the detection and management of cardiac allograft vasculopathy in transplant recipients. Clin Cardiol 2001; 24: 459-462.

5. Raff GL, Gallagher MJ, O’Neill WW, Goldstein JA. Diagnostic accuracy of noninvasive coronary angiography using 64-slice spiral com- 
puted tomography. J Am Coll Cardiol 2005; 46: 552-557.

6. Schlosser T, Mohrs OK, Magedanz A, Voigtlander T, Schmermund A, Barkhausen J. Assessment of left ventricular function and mass in patients undergoing computed tomography (CT) coronary angiography using 64-detector-row CT: comparison to magnetic resonance imaging. Acta Radiol 2007; 48: 30-35.

7. Achenbach S, Ropers D, Kuettner A, Flohr T, OHNESORGE B, BRUDER $\mathrm{H}$ et al. Contrast-enhanced coronary artery visualization by dual-source computed tomography-initial experience. Eur J Radiol 2006; 57: 331-335.

8. Miller LW, Schlant RC, Kobashigawa J, Kubo S, RENLUND DG. 24th Bethesda conference: Cardiac transplantation. Task Force 5: Complications. J Am Coll Cardiol 1993; 22: 41-54.

9. Bashore TM, Bates ER, Berger PB, Clark DA, Cusma JT, Dehmer GJ et al. American College of Cardiology/Society for Cardiac Angiography and Interventions Clinical Expert Consensus Document on cardiac catheterization laboratory standards. A report of the American College of Cardiology Task Force on Clinical Expert Consensus Documents. J Am Coll Cardiol 2001; 37: 2170-2214.

10. Leschka S, Alkadhi H, Plass A, Desbiolles L, GrunENFELDER J, MARINCEK B et al. Accuracy of MSCT coronary angiography with 64-slice technology: first experience. Eur Heart J 2005; 26 : 1482-1487.
11. Sigurdsson G, Carrascosa P, Yamani MH, Greenberg NL, Perrone S, Lev G et al. Detection of transplant coronary artery disease using multidetector computed tomography with adaptative multisegment reconstruction. J Am Coll Cardiol 2006; 48: 772-778.

12. Gregory SA, Ferencik M, Achenbach S, Yeh RW, HoffMANn U, IngLEssis I et al. Comparison of sixty-four-slice multidetector computed tomographic coronary angiography to coronary angiography with intravascular ultrasound for the detection of transplant vasculopathy. Am J Cardiol 2006; 98: 877-884.

13. Ferencik M, Gregory SA, Butler J, Achenbach S, Yeh RW, Hoffmann U et al. Analysis of cardiac dimensions, mass and function in heart transplant recipients using 64-slice multi-detector computed tomography. J Heart Lung Transplant 2007; 26: 478-484.

14. Romeo G, Houyel L, Angel CY, Brenot P, Riou JY, PAUL JF. Coronary stenosis detection by 16-slice computed tomography in heart transplant patients: comparison with conventional angiography and impact on clinical management. J Am Coll Cardiol 2005; 45: 1826-1831.

15. Bastarrika G, Arraiza M, De Cecco CN, MastroBUONI S, Ubilla M, Rábago G. Quantification of left ventricular function and mass in heart transplant recipients using dual-source CT and MRI: initial clinical experience. Eur Radiol 2008; 18: 1784-1890. 
